Vol.20,\#7, Junio 2011

http://revista-redes.rediris.es

\title{
Las asociaciones en las redes personales. ¿Mecanismo de integración de la población inmigrante?*
}

\author{
Mireia Bolíbar - Centre d’Estudis Sociològics sobre la Vida Quotidiana i el \\ Treball (QUIT), Departamento de Sociología, UAB ${ }^{1}$
}

\begin{abstract}
Resumen
Este artículo tiene como objetivo presentar algunos datos que permitan avanzar en el conocimiento de las funciones relacionales del asociacionismo entre la población inmigrante en Catalunya. Incide de lleno en el debate que confronta las teorías de la integración, las cuales destacan el círculo virtuoso de las asociaciones y su potencial para el desarrollo de capital social más allá del interior del grupo, respecto aquellas otras teorías que consideran las asociaciones como instituciones que refuerzan la homofilia y la creación de estructuras paralelas sin contacto con las existentes, que facilitan la creación y reproducción del capital social bonding. Confrontamos con estas teorías los datos de redes personales obtenidos en el marco del proyecto de investigación en curso CASREDIN. Se pone especial énfasis en mostrar la composición de las redes (en lo que respecta al origen y el lugar de residencia de los Alteri que conforman la red) y el apoyo social que se obtiene de ellas a partir de la participación en asociaciones, tanto de forma directa como indirecta. Asimismo, en este artículo se exploran las diferencias entre diversos tipos de asociación con el fin de comparar sus efectos sobre la composición y estructura de las redes.
\end{abstract}

Palabras clave: Asociacionismo - Inmigración - Capital social - Integración social - Redes sociales egocentradas - Apoyo social.

\begin{abstract}
This article aims to present some data about the relational functions that associations have among migrant population. Thus, entails to study the debate that confronts integration and homophily theories. The first ones highlight the virtuous circle of associations and their potential for the development of social capital beyond social groups. Whereas, the second ones consider associations as institutions that reinforce homogeneity on social networks and create parallel structures, creating and reproducing bonding social capital. Both theories are confronted with data of personal networks from the project CASREDIN. The results of this research show direct and indirect effects of participation in associations regarding to the composition of their networks (in terms of origin and place of residence of their Alters) as well as the social support obtained from them. Moreover, differences between various types of association are also explored.
\end{abstract}

Key words: Associationism - Migration - Social Capital - Social integration Egocentered social networks - Social support.

\footnotetext{
* Este artículo proviene de una investigación que tiene como referencia CSO2008-01470, subvencionada por el MiCINN dentro del marco del VI Plan Nacional de Investigación Científica, Desarrollo e Innovación Tecnológica 2008-2011.

${ }^{1}$ Enviar correspondencia a Mireia.Bolibar@uab.cat. Centre d’Estudis Sociològics sobre la Vida Quotidiana i el Treball (QUIT). Departament de Sociologia. Universitat Autònoma de Barcelona.
} 


\section{Introducción}

Una asociación es un grupo de personas u organizaciones formalmente organizado y con un nombre, que participan voluntariamente sin una recompensa económica por su participación (Knoke, 1986; en Montero, Font y Torcal (eds.), 2006).

Según Casey (1997) a medida que evoluciona el asentamiento del colectivo de inmigrantes evoluciona también la formación de asociaciones, pasando de ser puntos de encuentro informales a entidades formales y bien estructuradas con recursos y capacidad de movilización. Paralelamente a la formación de asociaciones de y por inmigrantes también se han ido desarrollando asociaciones para los inmigrantes (Colectivo Ioé, 1987); grupos de solidaridad y apoyo a este colectivo, mayormente creadas por población autóctona, así como otras organizaciones ya existentes que en su trabajo con población en riesgo de exclusión o desfavorecida socialmente han orientado su trabajo hacia el colectivo inmigrante.

Estas formas de participación han captado la atención de políticos, técnicos y organizaciones que trabajan en el ámbito de la intervención social, que ven en ellas un potencial importante para la integración de la población inmigrante, y buscan tener interlocutores que representen a determinados grupos de población, por lo que elaboran planes y proyectos para promover el asociacionismo entre este colectivo ${ }^{2}$.

Por otro lado, en el ámbito de las ciencias sociales, a raíz de la publicación de Putnam de 1993 se han generado un gran número de estudios relacionados con el asociacionismo, el capital social y sus relaciones mutuas, en algunos casos equiparando genéricamente el concepto de capital social a la misma participación en asociaciones, y sobretodo enfocados a estudiar las consecuencias de esta participación sobre el fortalecimiento de la democracia y el desarrollo económico.

Este artículo plantea un estudio desde el análisis de redes sociales para conocer cuál es el impacto de la pertenencia y participación en asociaciones sobre el proceso de integración (en el plano de las relaciones interpersonales) de la población inmigrante en la sociedad de acogida.

Se parte de la idea de que el estudio de las redes sociales egocentradas es una herramienta para obtener información no solo de los Egos entrevistados, sino sobre

\footnotetext{
2 El "Pacte Nacional per a la Immigració. Un pacte per viure junts i juntes" de 2008 incorpora varias medidas para promover el asociacionismo e incorporación de la población inmigrada en asociaciones ya existentes en Catalunya.
} 
el conjunto de la sociedad que condiciona la configuración concreta de la red social. Las redes personales son el resultado de las fuerzas que reproducen la sociedad: la organización social y económica, las instituciones culturales y las acciones intencionadas y los resultados previstos (o no) de sus individuos (Giddens 1967, en Molina et.al., 2008). Su composición, estructura y patrón de cambio se pueden considerar como una imprenta de las fuerzas que mueven la sociedad, así como el espacio de creación de cada individuo y las dinámicas emergentes debidas al azar o la transitividad en las relaciones (Molina et.al., 2008: 51).

\section{Los conceptos de integración y cohesión social desde la perspectiva del capital social}

El concepto de integración social es un concepto multidimensional ampliamente trabajado desde las ciencias sociales. En este artículo recogemos la elaboración teórica realizada por Lozares et al. (2011, en este monográfico), para aplicarlo a nuestro objeto de estudio particular. Concretamente nos centramos en estudiar la integración relacional de la población inmigrante, es decir, la integración como forma de distribución de las relaciones del colectivo de población inmigrada en Catalunya (España) con otros colectivos y con el conjunto de la población.

Siguiendo la conceptualización de Lozares (2003, 2006, 2008), entendemos que el capital social es un recurso social proveniente de las interacciones sociales entre dos entidades sociales (individuos, organizaciones, grupos, etc.). El capital social es dual: se construye, se expresa y se distribuye a través de la red que forma su estructura (la forma), al mismo tiempo que tiene una identidad sustantiva que puede ser diversa según los contenidos de las relaciones consideradas. Además, pueden producirse tres formas generales de orientación de las relaciones o formas de estructuración y distribución del capital social, que expresan de forma reticular los fenómenos de la Cohesión, la Vinculación y la Integración sociales.

La cohesión social se expresa en los lazos bonding, relaciones hacia dentro de un colectivo, y horizontales entre los miembros de un mismo grupo. La operativización de este concepto vincula la cohesión con la presencia de contactos homofílicos en la red, que comparten con Ego la pertenencia a un grupo o categoría social parecida (por ejemplo, personas del mismo colectivo de origen). En este artículo dejamos de lado los indicadores de cohesión de la estructura de la red (como por ejemplo la densidad, proximidad, etc.), centrándonos en su composición. La vinculación social se equipara al capital social bridging, los vínculos horizontales orientados al exterior de un grupo dado (relaciones entre, inter, hacia fuera o externas), como por 
ejemplo las relaciones que mantiene una persona inmigrante con otras personas también inmigrantes pero de un colectivo de origen diferente al propio. $Y$ finalmente, la integración social se observa en las formas de distribución del capital social de tipo linking, vínculos orientados hacia el exterior de un colectivo y verticales (entre pares de entidades de diferente envergadura y/o naturaleza, es decir, con diferencias de poder, prestigio, extensión o recursos; o bien de un individuo o colectivo - una parte- con el conjunto -la totalidad-). En esta investigación operativizamos este concepto equiparándolo, en el caso de la población inmigrante, a las relaciones y vínculos con personas autóctonas de la sociedad de acogida.

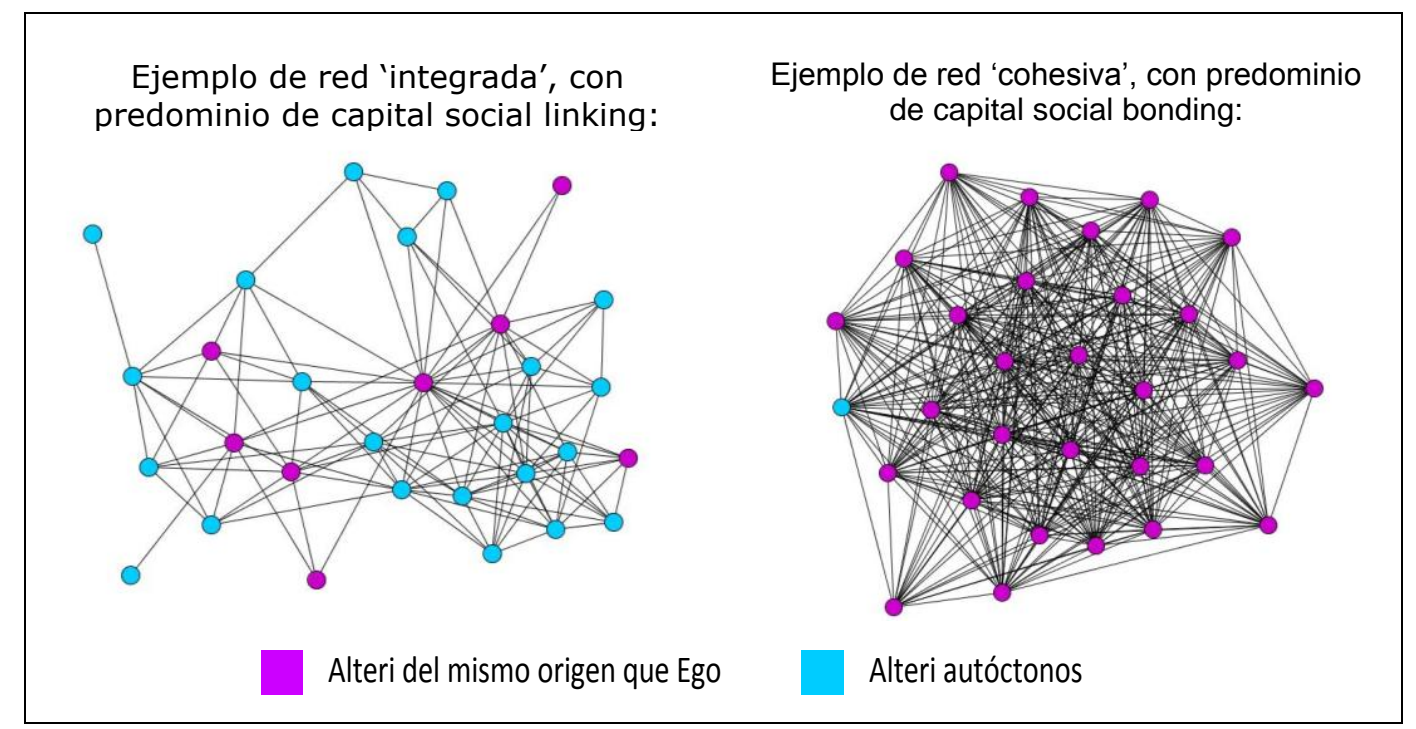

Figura 1. Casos de estudio que muestran la diversidad de formas de distribución del capital social. Fuente: Elaboración propia.

Esta perspectiva o forma de conceptualizar el capital social nos permite hacer uso del análisis de redes sociales y preguntarnos sobre las dinámicas de integración, vinculación y cohesión sociales de la población inmigrante en las sociedades de acogida mediante el estudio de casos comparativo de sus redes de relación interpersonal (como por ejemplo en la Figura 1). Además, la contextualización de estos casos y sus redes en organizaciones del tejido asociativo puede ayudarnos a estudiar las especificidades relacionales en este campo social también en referencia a la integración y la cohesión sociales. 


\section{El impacto de la participación en asociaciones en las redes sociales de la población inmigrante}

Existe un acuerdo en la literatura existente sobre el hecho que las asociaciones son una fuente de recursos relacionales. El mundo asociativo es un ámbito de socialización con la comunidad local (Wollebaék y Selle, 2004; en Prakash y Selle (Eds.)). Formar parte de una asociación, en la gran mayoría de casos, implica "meterse" en un grupo de personas, ampliar la red social y conocer gente. En el caso de la población inmigrante, además, en un contexto de ruptura y cambio del entorno y de la red de contactos, el asociacionismo permite superar el aislamiento social en el proceso de asentamiento de las personas inmigradas en la sociedad de acogida (Morell 2005), así como fomentar la sociabilidad y el intercambio de experiencias (Ribas 2003, en Garreta et.al. 2008).

\section{La red de apoyo como fuente de recursos: las redes informales nacidas de la institucionalización formal}

Un foco importante de la literatura existente sobre el asociacionismo inmigrante destaca su importancia en la generación de una red de apoyo, y en la generación de redes que amplían las fuentes informales de apoyo social de quienes participan en ellas. El concepto de apoyo social se refiere a los recursos afectivos e instrumentales que se obtienen en la interacción con los demás (Maya, Martínez y García, 1999). El apoyo social es uno de los contenidos funcionales de las relaciones sociales, que hace referencia a aspectos positivos de las relaciones, siendo la "red de apoyo social" el subconjunto de la red personal del individuo que proporciona información, ayuda instrumental y apoyo afectivo (Maya, 2006).

Algunas reflexiones en torno al fenómeno del asociacionismo inmigrante destacan su papel en el campo afectivo. Para Morell (2005), las asociaciones responden a un "imperativo afectivo": a la necesidad humana de encontrarse, reunirse, dialogar, intercambiar efectos, inquietudes y experiencias, etc.; limitando un marco de pertenencia que proporciona seguridad (Lluch, 1998). Otras, en cambio, destacan su vertiente instrumental del apoyo. Según Sipi (2000), las asociaciones nacieron con el objetivo, entre otros, de sostener redes de solidaridad. Del mismo modo Garreta (1998) argumenta que uno de los principales objetivos que persiguen las asociaciones de inmigrantes es la de crear y potenciar una red de relaciones entre los residentes que actúe como protección ante situaciones de necesidad. 
Además, no es sólo el apoyo intercambiado con gente de un origen similar, sino también con la gente de la cultura de recepción la que mejor predice una integración social exitosa (Berry, 1997; en De Miguel Luken y Tranmer, 2010). La existencia de vínculos con autóctonos en la red personal de apoyo, puede también ser considerada como un indicador clave de la integración de los inmigrantes (Martínez García et. al., 2001), al mismo tiempo que tiene importancia por su contenido substantivo (de Miguel Luken, Tranmer, 2010).

\section{La teoría de la integración vs. la teoría del enclave}

En esta línea, existe un debate sobre cuál es el impacto de la participación en asociaciones sobre las redes de la población inmigrante. Siguiendo la clasificación de Glanville (2004) y Lauer y Yan (2010) distinguimos entre las teorías que podemos englobar dentro de las "teorías de la integración", que destacan el círculo virtuoso de las asociaciones y su potencial para el desarrollo de capital social más allá del interior del grupo. Y por otro lado, las "teorías del enclave", las cuales consideran las asociaciones como instituciones que refuerzan la homofilia y la creación de estructuras paralelas sin contacto con las existentes, que facilitan la creación y reproducción del capital social bonding.

La hipótesis de la integración sostiene que la participación en asociaciones promueve la diversidad en las redes personales. Esta tesis, ampliamente difundida por Putnam, enfatiza genéricamente el potencial de las asociaciones para crear puentes entre mundos sociales diferentes (Putnam, 2000; Baldassari y Diani, 2007), y crear una cultura cívica que permita el intercambio y la convivencia dentro de la diversidad (Rodríguez-García, 2010; Putnam 2007). Según Castles y Miller (1994, en Morell 2005), las asociaciones voluntarias son una manifestación necesaria del asentamiento de un grupo, que facilita la negociación de su participación social e integración afectiva en la sociedad de acogida. En el marco del proceso migratorio, el asociacionismo fomenta la sociabilidad, el intercambio de experiencias, y disponer de referentes de integración (Morell, 2005). La contribución de las asociaciones a la integración social radica en su capacidad de crear redes múltiples y superpuestas capaces de reducir el potencial conflictivo de una sociedad (Wollebaek y Selle, en Prakash y Selle (eds.), 2004), proveyendo oportunidades de interacción entre diferentes segmentos de una comunidad, y consecuentemente proporcionando oportunidades para reducir fracturas (cleavages) que crean conflicto, inequidad y desconfianza (Babchuk y Edwards, 1965; Olsen, 1982; en Glanville, 2004). 
Por otro lado, las teorías del enclave, sostienen que la participación en asociaciones voluntarias comporta un repliegue étnico, incrementa la homofilia y la cohesión relacional. Desde esta perspectiva se ha mostrado cómo las asociaciones proporcionan oportunidades de interacción entre individuos similares, incrementando así la similitud de las redes sociales. Estudios anteriores han señalado la homogeneidad en los entornos asociativos en términos de edad, clase, religión, etnia y género (Feld, 1982; Marsden, 1990; McPherson y Smith-Lovin 1987; y Popielarz y McPherson, 1995). Por ejemplo, Rotolo (2000) o Marsden (1990) han mostrado como la pertenencia en asociaciones es más común en barrios con mayor homogeneidad racial y entre personas con una red más homogénea en términos religiosos (Bekkers et al, 2008). También según Molina, Lerner y Gómez (2008: 59), analizando los patrones de cambio de las redes personales de inmigrantes en Catalunya, las asociaciones producen un efecto "involutivo", en el sentido de incrementar la homogeneidad y la endogamia; con la excepción de las personas que desempeñan un rol directivo en dichas asociaciones, que se relacionan con administraciones, sindicatos, ONGs y partidos políticos. Este fenómeno es lo que ha llevado a hablar de un posible efecto de "guetización asociativa" de la minoría (Morell 2005), institucionalizando estructuras paralelas a las existentes en la sociedad de acogida (Garreta, 1998).

Merece la pena señalar que nos encontramos con una limitación en la generación de afirmaciones causales en relación al impacto de participar en asociaciones sobre las redes sociales de sus participantes. El uso de datos cuantitativos nos permite establecer asociaciones y coexistencias entre variables y fenómenos sociales, pero no establecer su causalidad de forma segura. Así, hemos interpretado los datos partiendo de la idea que la pertenencia asociativa puede provocar un cambio en la configuración de las redes de sus participantes, aunque ya apuntamos que no podemos descartar que se produzcan otros efectos. Por ejemplo, que san ciertos individuos, con un perfil relacional específico quienes tiendan más a participar en asociaciones, o incluso que ciertas características atributivas de ellos sean las que marquen un patrón en sus relaciones a la vez que les impulsen a participar.

\section{La exploración de los datos}

\section{El planteamiento metodológico}

El trabajo empírico de este estudio se realiza con los datos de la investigación en curso CASREDIN: "Estudio comparado de casos sobre la influencia mutua entre capital e integración sociales y la inserción, estabilidad, promoción y cualificación en el empleo", proyecto I+D+I con referencia CSO2008-01470, subvencionado por 
el Ministerio de Educación y Ciencia dentro del marco del VI Plan Nacional de Investigación Científica, Desarrollo e Innovación Tecnológica 2008-2011, y el investigador principal del cual es Carlos Lozares. En el artículo "El análisis de la cohesión, vinculación e integración sociales en las encuestas Ego-net" (Lozares et. al., 2011), en este mismo monográfico, se exponen los detalles de su planteamiento y diseño. Se trata de una encuesta realizada durante el 2009 y 2010 a partir de una muestra aleatoria, estratificada por cuotas y bietápica. La ciudad de residencia de los encuestados (tres ciudades de diferente envergadura que recogen en buena medida la variabilidad urbana del territorio catalán), y el colectivo de origen (comprendido en base al lugar de nacimiento, el de los padres y la edad) han sido los criterios de estratificación. Concretamente, se han recogido 450 encuestas egocentradas de los siguientes colectivos: catalanes -nacidos en Catalunya- de entre 25 y 55 años con padres catalanes, así como de más de 55 años; catalanes con padres nacidos en el resto de España de entre 25 y 55 años; mayores de 55 años nacidos en el resto de España; Ecuatorianos - nacidos en Ecuador-; y Marroquíes - nacidos en Marruecos-. Los datos informan de las características atributivas de los Egos, de los Alteri (obtenidos a través de un generador flexible de nombres), de la relación Ego-Alter, y de la relación AlterAlter.

Las variables de la base de datos referidas específicamente a la participación informan sobre la pertenencia asociativa de los Egos (si participan o no en alguna asociación y en cuantas, el tipo de asociación y el grado de implicación en ellas) así como de si los Alteri nombrados en la red forman parte de la misma asociación de Ego. La forma de preguntar sobre la participación en asociaciones en esta encuesta (enumerando de una a una posibles tipos diferentes de asociación) tiene resultados muy positivos para hacer emerger formas asociativas que de otro modo quedan ocultas en el momento de la encuesta (San Martín, 2009). Este factor, sumado a las particularidades del trabajo de campo y las estrategias utilizadas para contactar con los encuestados ha resultado en una sobrerrepresentación de la población asociada. La base de datos utilizada cifra en el $55 \%$ la proporción de población inmigrante que participa, un $10 \%$ más de lo que se detalla en la 'Enquesta de condicions de vida i hàbits de la población de Catalunya' de 2006.

La exploración del impacto de la pertenencia asociativa que se propone en este artículo (en este caso, sobre las redes de los migrantes en Catalunya -España-) aporta elementos novedosos respecto las investigaciones anteriores ya que, en primer lugar, explora redes egocentradas con 30 Alteri, $y$, en segundo lugar, aporta datos sobre la composición (quienes componen las redes y cuales son sus 
características atributivas), sobre la estructura (cómo son las estructuras a las que dan forma las relaciones entre los Alteri de un Ego), y sobre el contenido de las redes (cómo es la relación entre Ego y cada uno de los Alter, qué recursos se intercambian, etc.) de forma muy extensa y completa.

Concretamente, los elementos clave que tenemos en cuenta son el origen étnico o migratorio de los Alteri que configuran las redes; su ubicación espacial; la temporalidad de las relaciones; la estructura de las redes y el intercambio de apoyo social. El procedimiento de análisis se basa en la comparación de los elementos de las redes explicitados según las siguientes características de los Egos encuestados: El origen (autóctonos vs. inmigrantes), la participación en asociaciones (asociados/as vs. no asociados/as), y el tipo de asociación en el que se participa. También comparamos la sub-red de Alteri que participan en la misma asociación de Ego respecto el resto de la red.

\section{La dinámica relacional en el tejido asociativo: ¿dinámicas propias?}

Los datos muestran que la sociedad catalana en el plano de las redes de relación personal es una sociedad bastante fragmentada y cerrada en relación al origen (étnico o migratorio). Aplicando los indicadores de medición de la cohesión e integración sociales que tratan de capturar los conceptos anteriormente citados (detallados en Lozares et. al. 2011, en este monográfico), observamos como en todos los colectivos la cohesión social supera a la integración social en el balance entre ambas. Los índices que se muestran en estas tablas expresan una ratio (algo modificada), para cada colectivo de Egos, entre aquellos Alteri atributivamente semejantes (en este caso, en lo que atañe al origen) respecto aquellos Alteri diferentes a los Egos con los que se relacionan. De este modo, cuanto más cercano a 1 se encuentra el índice de balance, más equilibradas están las redes de los Egos, mientras que cuanto más elevado sea el índice, más cohesivas son en lo que respecta a la composición de la red (en relación a la variable que se considere). Los índices de balance de las diferentes tablas son comparables entre ellos (siempre y cuando se tengan en cuenta el mismo número de categorías de Egos y Alteri) ${ }^{3}$.

\footnotetext{
${ }^{3}$ Concretamente este índice expresa el porcentaje de Alteri del mismo origen que Ego dividido entre la media de los porcentajes de Alteri del mismo origen que el resto de los Egos contemplados. De este modo, el índice es sensible al número de categorías de Egos y Alteri que se tengan en cuenta. Por este motivo cuando sólo trabajamos con las categorías "autóctonos e inmigrantes" -en las tablas 2, 4 y $7-$ el índice se mueve en números más bajos, mientras que cuando se tiene en cuenta los Egos de origen de base de la investigación CASREDIN (que contempla el lugar de nacimiento, el factor generacional, etc.), -en las tablas 1 y 6 - el denominador es más bajo y por tanto se trabaja con números más altos.
} 


\begin{tabular}{|c|c|c|c|c|c|c|c|}
\hline \multirow[b]{2}{*}{ Balances } & \multicolumn{6}{|c|}{ Origen Ego } & \multirow[b]{2}{*}{ Cramer* } \\
\hline & $\begin{array}{c}\text { Nacidos } \\
\text { Catalunya } \\
\text { padres } \\
\text { Catalunya } \\
\text { +55 años }\end{array}$ & $\begin{array}{c}\text { Nacidos } \\
\text { Catalunya } \\
\text { padres } \\
\text { Catalunya } \\
25-55 \\
\text { años }\end{array}$ & $\begin{array}{c}\text { Nacidos } \\
\text { Catalunya } \\
\text { padres } \\
\text { España } \\
25-55 \\
\text { años }\end{array}$ & $\begin{array}{c}\text { Nacidos } \\
\text { resto } \\
\text { España } \\
+55 \text { años }\end{array}$ & Ecuador & $\begin{array}{l}\text { Marrue- } \\
\text { cos }\end{array}$ & \\
\hline $\begin{array}{c}\text { Total } \\
\text { población }\end{array}$ & 4,62 & 9,34 & 3,02 & 5,54 & 9,29 & 16,27 & 0,576 \\
\hline $\begin{array}{l}\text { Población } \\
\text { asociada }\end{array}$ & 4,56 & 9,83 & 2,54 & 6,62 & 7,78 & 15,23 & 0,578 \\
\hline $\begin{array}{c}\text { Población } \\
\text { no asociada }\end{array}$ & 4,96 & 8,02 & 3,62 & 4,07 & 11,10 & 18,23 & 0,565 \\
\hline
\end{tabular}

*Intensidad de la relación Origen Ego X Origen Alter. (Sig. = 0,000). Fuente: elaboración propia.

Tabla 1. Índices del balance cohesión-integración sociales (por categorías de origen) para el conjunto de la población y separadamente para la población asociada y no asociada, en función del origen de los Egos.

La tendencia a la cohesión de las redes sobre los colectivos de origen es mayor entre los colectivos de inmigrantes, especialmente el colectivo marroquí, seguido, en orden decreciente, por el colectivo de catalanes jóvenes (entre 25 y 55 años), el de mayores nacidos en el resto de España, el de catalanes mayores (de más de 55 años) y finalmente el de jóvenes nacidos en Catalunya de padres nacidos en el resto de España, los cuales tienen unas redes más equilibradas en el balance cohesión-integración sociales.

Sentada esta premisa como tendencia general, la comparación de los balances entre la población asociada y no asociada (tabla 1) muestra como, en el caso de la población inmigrante, el desequilibrio a favor de la cohesión es menos pronunciado entre aquellos que participan en asociaciones respecto los que no participan en ellas. En cambio, entre la población autóctona hay también diferencias internas: en el caso de los colectivos de catalanes mayores y nacidos en Catalunya de padres nacidos en el resto de España sucede lo mismo que entre los inmigrantes (es decir, la población no asociada tiene unas redes más cohesivas), mientras que en el caso de los catalanes jóvenes y los españoles mayores sucede lo contrario: las personas asociadas tienen redes más cohesivas que las no asociadas.

Un recálculo de los índices aplicado al análisis de la relación entre Egos y Alteri de las categorías generales de autóctonos e inmigrantes (tabla 2) expone un resultado diferente a lo sugerido hasta ahora: La población autóctona tiene unas redes mucho más cohesivas y cerradas sobre sí mismas que la población inmigrante, y, lo que nos interesa más para el contraste de las hipótesis, la población inmigrante asociada dispone de unas redes de relación interpersonales más cohesivas y menos integradas que la población inmigrante no asociada. Asimismo, la población 
autóctona que participa en asociaciones tiene más vínculos con personas inmigrantes que los no asociados.

\begin{tabular}{|c|c|c|c|}
\hline \multirow{2}{*}{ Balances } & \multicolumn{2}{|c|}{ Origen Ego } & \multirow{2}{*}{$\begin{array}{c}\text { V } \\
\text { Cramer* }\end{array}$} \\
\hline & Autóctono & Inmigrante & \\
\hline Total población & 28,39 & 4,06 & 0,798 \\
\hline Población asociada & 27,83 & 4,34 & 0,805 \\
\hline Población no asociada & 29,68 & 3,75 & 0,784 \\
\hline
\end{tabular}

*Intensidad de la relación Origen Ego X Origen Alter $(\mathrm{Sig} .=0,000)$. Fuente: elaboración propia.

Tabla 2. Índices del balance cohesión-integración sociales (por categorías autóctono-inmigrante) para el conjunto de la población y separadamente para la población asociada y no asociada.

Aunque parezcan contradictorios, lo que los datos presentados parecen señalar es que la población inmigrante asociada, respecto la no asociada, dispone de unas redes de relación interpersonales menos cerradas hacia el propio grupo de origen "nacional" (con personas del mismo país de nacimiento que Ego) pero un poco más cerradas en el colectivo de inmigrantes (nacidos en el extranjero) en general. Así, entre las redes de las personas que participan en el campo asociativo cobran mayor importancia los vínculos bridging, de vinculación horizontal con otros grupos de inmigrantes. No sólo el mayor porcentaje de Alteri de otros orígenes con los que los Egos tienen relación; también la aplicación del índice de Vinculación -Vns2- a los colectivos de origen base de la investigación (Lozares et al 2011, en este monográfico) refuerza esta realidad: mientras que dicho índice para los no asociados es de 0, para los asociados es de 0,004 (aunque esta tendencia es debida especialmente a los ecuatorianos, para quienes el índice de vinculación entre los asociados/as es del 0,125$)$.

En la exploración de los datos también se ha observado una diferencia significativa en el impacto de la participación sobre la integración relacional según el sexo de Ego. A diferencia de lo que describe Hagan (1998) sobre las mujeres inmigrantes maya en Estados Unidos, quienes participan en pequeños grupos de mujeres que limitan su capacidad para desarrollar redes con personas no co-étnicas, en el caso de las mujeres ecuatorianas y marroquíes en Catalunya más bien parece que aquellas que participan en asociaciones tienden a desarrollar estos vínculos en mayor medida que las que no participan. Así, la comparación de las medias del porcentaje de Alteri extranjeros presentes en la red de aquellos individuos que no participan en asociaciones muestra una clara diferencia (significativa estadísticamente al 0.002) en la integración relacional de hombres y mujeres; mientras que esta diferencia se atenúa (dejando de ser significativa) entre los hombres y las mujeres que sí participan en asociaciones. Entre los hombres 
presentes en el ámbito asociativo se reduce la proporción de vínculos con autóctonos que poseen, mientras que entre las mujeres aumenta.

\begin{tabular}{|c|c|c|}
\hline \multirow{2}{*}{} & \multicolumn{2}{|c|}{ Sexo de Ego } \\
\cline { 2 - 3 } & $\hat{1}$ & $\emptyset$ \\
\hline $\begin{array}{c}\text { Ego NO participa } \\
\text { en asociaciones }\end{array}$ & $71,72 \%$ & $86,67 \%$ \\
\hline $\begin{array}{c}\text { Ego SÍ participa } \\
\text { en asociaciones }\end{array}$ & $80,24 \%$ & $82,39 \%$ \\
\hline
\end{tabular}

Tabla 3. Comparación del porcentaje medio de Alteri inmigrantes presentes en las redes de los Egos en función del sexo y la participación en asociaciones. Fuente: elaboración propia.

Molina et.al. (2008) en el estudio de la evolución de las redes de los inmigrantes realiza una distinción interesante entre los Alteri presentes en las redes en función de su lugar de residencia. A este eje de análisis le añadimos otra distinción interesante a tener en cuenta para entrar en la dinámica espacio-temporal de las relaciones: la diferenciación entre los Alteri en función del lugar en el que se inició la relación. De este modo, la distinción entre aquellos Alteri del mismo origen y residentes en el país de destino que se conocieron en el país de origen (Alteri anteriores al proceso migratorio) respecto los Alteri también del mismo origen y residentes en el país de destino que se conocieron en el país de destino (desafiando las leyes de la probabilidad, según las cuales estos deberían ser una minoría), nos permite entrever la existencia y creación de grupos étnicos fuertes en la sociedad catalana con el denominador común del compartir el origen. Observamos como, entre aquellos Egos que participan en asociaciones, el $60 \%$ de los Alteri del mismo origen residentes en el país de destino son fruto de una relación originada en el país de destino y el $40 \%$ en el origen, mientras que entre aquellas personas que no participan en asociaciones la relación es de $56 \%$ y $44 \%$ respectivamente.

Hasta este punto sólo hemos presentado datos distinguiendo genéricamente entre las redes de aquellos Egos que participan en asociaciones respecto los que no participan. Sin embargo, si nos fijamos, entre aquellos individuos que participan en asociaciones, exclusivamente en aquellos contactos con los que se comparte la pertenencia asociativa, observamos un fenómeno interesante (tabla 4). Entre la población autóctona prácticamente no varía el porcentaje de inmigrantes con los que se tiene relación. En cambio, entre la población inmigrante, si se tienen en cuenta sólo los Alteri de la red que también participan y forman parte de la asociación de Ego, es mayor la proporción de personas autóctonas que participan en la misma asociación de Ego $(23,1 \%)$ que no las que no participan $(17,8 \%)$. De 
este modo, el índice de balance cohesión-integración teniendo en cuenta las categorías generales autóctono-inmigrante es menor (menos cohesivo incluso que el del conjunto de la población inmigrante no asociada); igual que la concordancia entre el origen de Ego y el del Alter (observable a través de la $\mathrm{V}$ de Cramer).

\begin{tabular}{|c|c|c|c|}
\hline \multirow{2}{*}{ Balances } & \multicolumn{2}{|c|}{ Origen Ego } & \multirow{2}{*}{ V Cramer* } \\
\cline { 2 - 3 } & Autóctono & Inmigrante & \\
\hline $\begin{array}{c}\text { Sub-red de Alteri que pertenecen } \\
\text { a la misma asociación que Ego }\end{array}$ & 27,55 & 3,33 & 0,760 \\
\hline $\begin{array}{c}\text { Sub-red de Alteri que no participa } \\
\text { a la misma asociación que Ego }\end{array}$ & 27,90 & 4,51 & 0,810 \\
\hline
\end{tabular}

*Intensidad de la relación Origen Ego X Origen Alter $($ Sig. = 0,000). Fuente: elaboración propia.

Tabla 4. Índices del balance cohesión-integración sociales de la población inmigrante asociada en función de si los Alteri comparten la actividad asociativa de Ego o no.

Teniendo en cuenta la dimensión espacial, vemos como aquellos Alteri con los que se comparte la actividad asociativa son contactos locales y muy cercanos (el 99,6\% reside en el mismo país que Ego y el $89,9 \%$ reside en la misma localidad, entre los cuales el $35,7 \%$ reside en el mismo barrio). Prácticamente no se observan tampoco relaciones con personas del campo asociativo de los egos que residan en el país de origen ni en otros países. Además, destaca la elevada proporción de estos Alteri con los cuales, siendo su origen también ecuatoriano o marroquí, la relación se ha iniciado en el país de destino (tabla 5 ). Y en lo que respecta al tiempo de conocimiento o duración de la relación, se observa como estos Alteri se han generado en relaciones mucho más recientes, con una media de 6,76 años de conocimiento respecto a los 16,96 del resto de relaciones de la población inmigrante en conjunto. 


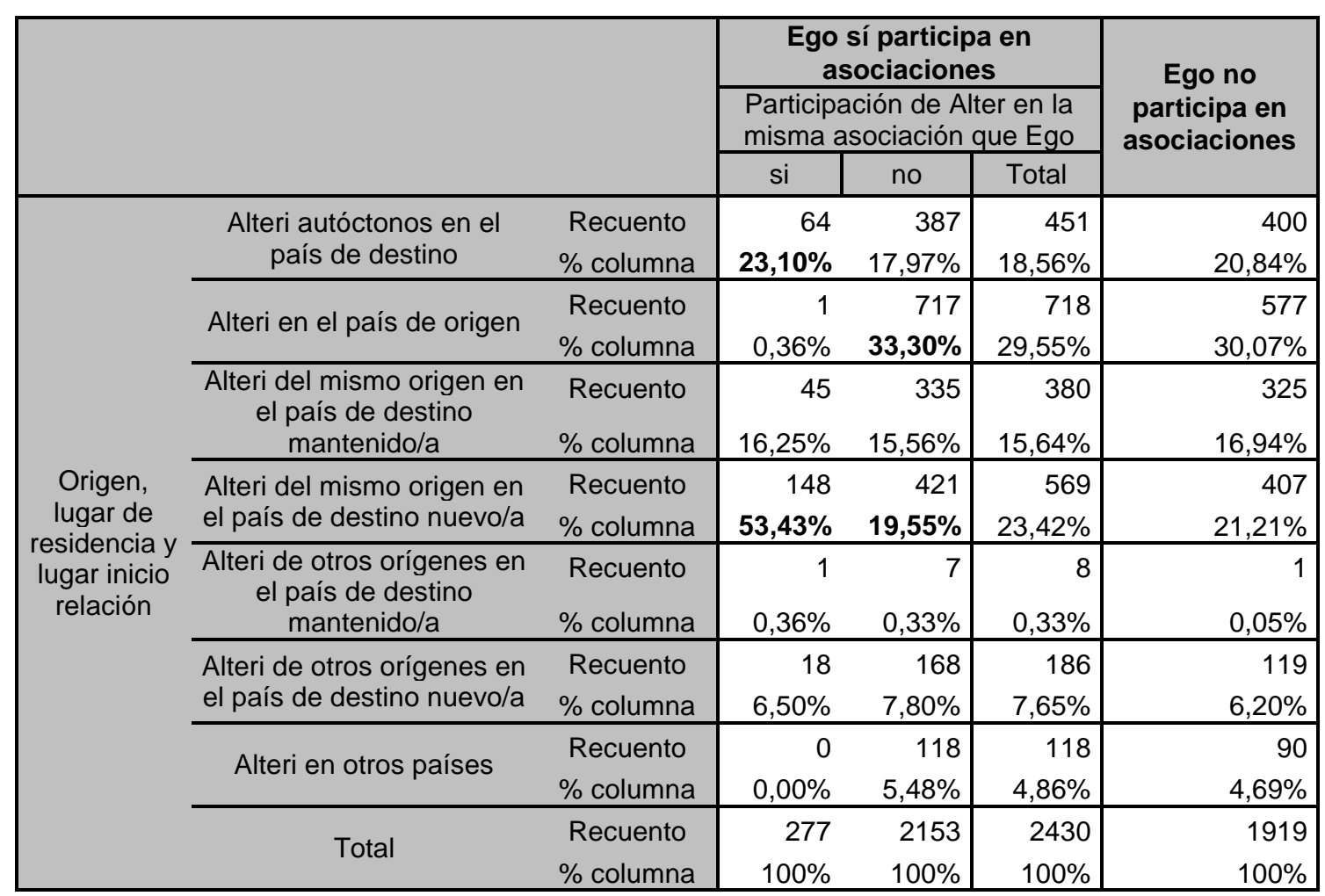

$\checkmark$ Cramer (Origen, lugar de residencia y lugar inicio relación X Participación de Alter en la misma asociación que Ego) $=0,307 ;$ Sig. = 0,000. Fuente: elaboración propia.

Tabla 5. Distribución de la composición de origen y espacio-temporal de las redes de la población inmigrante según si los Egos participan en asociaciones o no y, si es el caso, si los Alteri forman parte de la asociación de Ego.

En general, estos datos nos llevan a pensar que el hecho de que la población asociada tenga unas redes con menos capital social linking, integrador, no es debido propiamente al efecto directo de que las personas con las que se mantiene una relación por el hecho de pertenecer a la misma asociación tiendan más a ser inmigrantes. Se apunta al hecho de que las asociaciones tienen cierto efecto integrador entre la población inmigrante, pero que el impacto de este fenómeno sobre el conjunto de las redes de quienes participan en asociaciones no es suficientemente intenso como para que se observen cambios significativos en ellas (entre aquellos individuos que participan en asociaciones, sólo el 11,4\% de los Alteri participan en la misma asociación que Ego).

En lo que respecta a la estructura de las redes observamos como las personas asociadas disponen de redes con mayor presencia de subgrupos cohesivos. Así, estos Egos tienen, de media, 23 cliques en sus redes (Ncliques de 3 a distancia de 1), mientras que los Egos no asociados disponen de sólo 15. 
Finalmente, otra cuestión que hemos analizado es la obtención de apoyo social. Los datos señalan que las personas que forman parte de una asociación intercambian más apoyo social a través de sus redes personales. Concretamente, si comparamos una vez más las redes de las personas presentes en el mundo asociativo respecto las que no participan en ninguna asociación, observamos como las primeras afirman haber intercambiado ayuda con sus Alteri en un mayor número de ámbitos (distinguiendo entre los siguientes: laboral, educativo, sanitario, relacionado con la vivienda, problemas personales, servicios sociales y administrativos, u otros ${ }^{4}$ ). Teniendo en cuenta también el número de Alteri con los que se intercambia el apoyo social en estos ámbitos también podemos afirmar que las personas que participan en una asociación intercambian más apoyo con los Alteri de su red que las que no participan. Así, las primeras intercambian ayuda con el conjunto de los Alteri en 93,9 ocasiones, mientras que entre las que no participan la media de ocasiones es del 80,9.

Si nos fijamos más detenidamente en las personas en concreto que forman parte de la asociación en la que participan los asociados, también se observa como se intercambia apoyo en más ámbitos con estos Alteri que con el resto. Es especialmente significativa la obtención de apoyo a través de las personas que forman parte del entorno asociativo de los Egos en cuestiones relacionadas con el acceso a los servicios sociales y la solución de problemas administrativos.

\section{Nuevas aportaciones y matices: la introducción de la variable "tipo de participación"}

Estudios más recientes (Glanville 2004, Lauer y Yan, 2010) han empezado a matizar la dicotomización entre las teorías de la integración y las teorías del enclave y a resolver la inconsistencia de la literatura existente teniendo en cuenta la gran diversidad de asociaciones existentes y sus diferentes implicaciones para las redes sociales de los inmigrantes. Siguiendo a Lin (en Bekkers et.al., 2008), quien en 2001 argumentó que la distinción entre acción expresiva e instrumental podía ser usada para predecir efectos diferenciales en las redes (mientras que la acción expresiva se dirige a mantener los recursos, la acción instrumental se dirigiría a obtener otros nuevos), estos estudios parten de la diferenciación entre asociaciones instrumentales y expresivas con el fin de realizar un estudio comparativo del impacto de la pertenencia asociativa sobre las redes según el tipo de asociación que

\footnotetext{
${ }^{4}$ La pregunta de la encuesta en concreto se formula del siguiente modo: ¿Le ha proporcionado esta persona (o Ud. a ella), sea en la actualidad o en el pasado, informaciones útiles u otros medios o ayudas para... / encontrar o solucionar problemas de trabajo / acceder a servicios sociales y/o administrativos / superar problemas personales / etc. ...?
} 
se considere. Su forma de conceptualizar la participación instrumental o expresiva varía, pero ambos estudios concluyen que, en general, las organizaciones o la participación expresiva fomenta redes más homogéneas, mientras que la participación instrumental incrementa la diversidad de contactos personales que cruzan fronteras étnicas. En definitiva, estas investigaciones enfatizan la idea que el mundo asociativo no es una realidad monolítica sino diversa y plural, la cual cosa también se quiere tener en cuenta en el presente diseño de investigación.

En la Figura 1 se muestran los diferentes tipos de asociación a los que pertenecen los inmigrantes encuestados en la investigación CASREDIN ${ }^{5}$. Se observa cierta variabilidad interna en la composición de las redes (en la presencia de Alteri autóctonos) de los Egos en función del tipo de asociación al que pertenecen. Destacan especial y significativamente los extremos: quienes participan en ONG tienen (en promedio) el $24,7 \%$ más de autóctonos en sus redes que el resto de Egos, mientras que quienes participan en grupos u organizaciones para la inserción laboral tienen un $8,7 \%$ menos.

\footnotetext{
5 Los datos referentes al tipo de participación se han tratado construyendo variables de respuesta múltiple, con lo que aquellos casos que participan en más de un tipo de asociación se tienen en cuenta en todos ellos. Aunque este tratamiento provoque que sea más difícil entrever el "efecto neto" de la participación en un tipo concreto de asociación, permite ver en cada tipo de asociación el conjunto de individuos que participan en ella.
} 


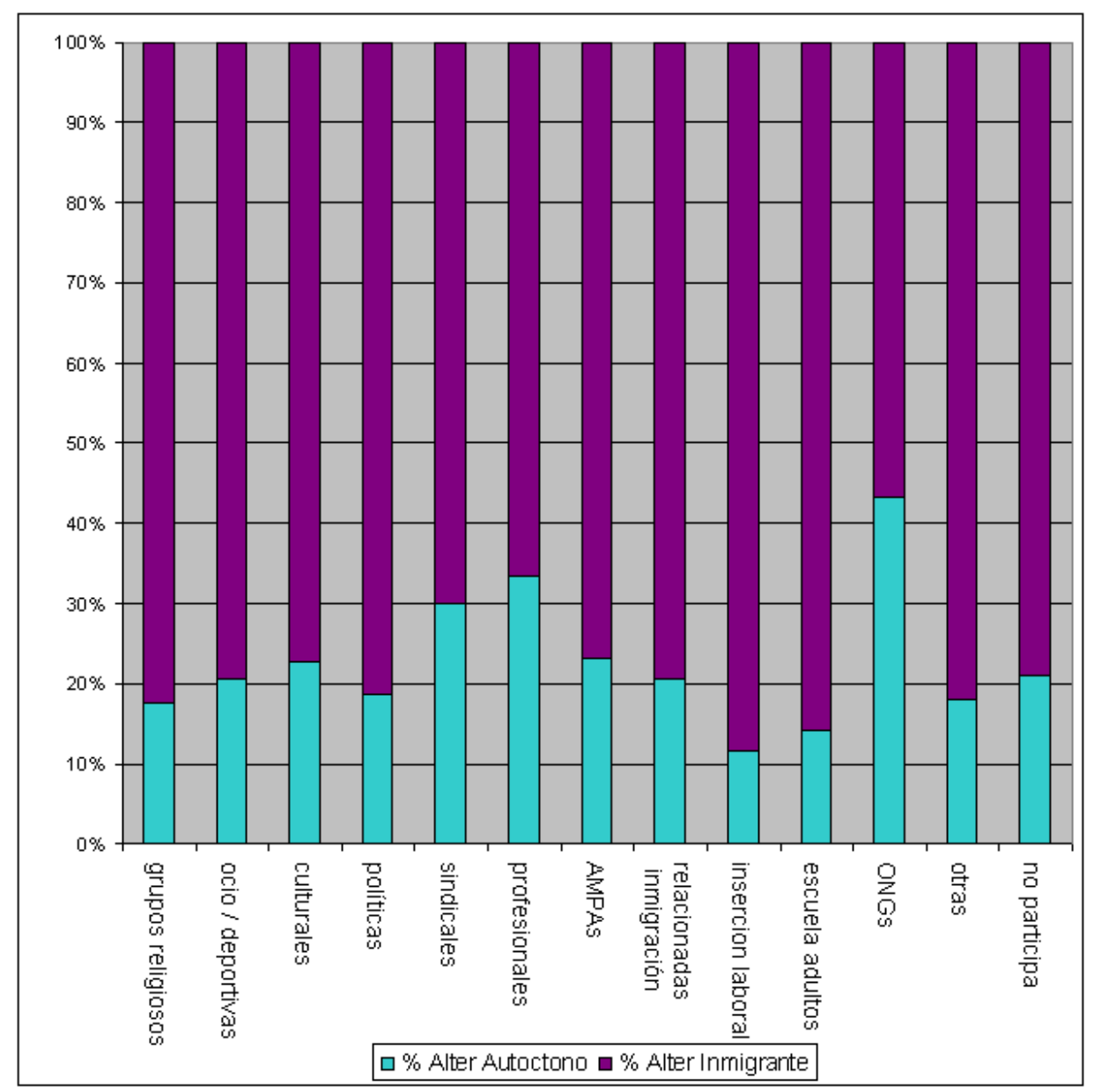

Figura 2. Caracterización del origen (autóctonos o inmigrantes) de los Alteri y la proporción de su presencia en las redes de los Ego según el tipo de asociación en el que participan los Egos. Fuente: elaboración propia.

A partir de los criterios teóricos presentes en la literatura y también de los datos presentados, hemos realizado una tipología de asociaciones. La tipología que hemos realizado tiene en cuenta, en primer lugar, la clásica distinción entre asociaciones expresivas e instrumentales (basada en la tipología de acción social de Max Weber que distingue entre la acción social basada en el sentimiento subjetivo, afectivo o tradicional, de pertenencia colectiva; de la acción social fundada en un acuerdo o compensación de intereses racionalmente motivados, intencionados y elegidos (Weber, 1978 en Bekkers et.al., 2008). A diferencia de la participación expresiva, la participación instrumental persigue unos objetivos o propósitos más allá del disfrute de la participación y la sociabilidad en si misma. Entre las asociaciones expresivas encontramos aquellos grupos y asociaciones religiosas, de ocio o deportivas y culturales. En cambio, entre las asociaciones instrumentales se engloban las políticas, sindicales, profesionales, las AMPAs y las ONGs. En segundo lugar también hemos tenido en cuenta la especificidad de aquellas organizaciones del tercer sector de 'voluntariado social' fuertemente orientadas a terceros (Ariño, 
2004), probablemente asociaciones para inmigrantes, y en las cuales los participantes más bien son usuarios de los servicios que estas ofrecen. Entre ellas encontramos las organizaciones para la inserción laboral y las escuelas de adultos, y las clasificamos como organizaciones "de servicios". Finalmente, también mantenemos la categoría propia de las asociaciones 'relacionadas con la inmigración', pues se trata de un tipo de asociación muy genérica y diversa, difícil de encajar en las otras tres categorías.

La aplicación de los índices de balance de la cohesión-integración a las redes de los encuestados en la investigación CASREDIN según la tipología elaborada de participación en asociaciones (tabla 6), del mismo modo que la comparación de los porcentajes promedio de extranjeros en las redes, señalan la especificidad de las redes de los participantes en asociaciones de servicios, siendo estas las más cohesivas y homofílicas. También observamos que entre los otros tres tipos de asociaciones hay menos variabilidad, aunque cabe destacar que los índices son más pequeños (por lo tanto, son redes menos cohesivas) entre quienes participan en asociaciones instrumentales.

\begin{tabular}{|c|c|c|c|c|c|}
\hline \multirow{3}{*}{$\begin{array}{l}\text { Balances } \\
\text { (inmigrantes } \\
\text { asociados } \\
\text { respecto } \\
\text { autóctonos) }\end{array}$} & \multicolumn{5}{|c|}{ Tipología participación en asociaciones } \\
\hline & $\begin{array}{c}\text { Instrumen } \\
\text { tal }\end{array}$ & Expresiva & $\begin{array}{c}\text { De } \\
\text { servicios }\end{array}$ & $\begin{array}{c}\text { Relacionadas } \\
\text { con la } \\
\text { inmigración }\end{array}$ & $\begin{array}{c}\text { No } \\
\text { participa }\end{array}$ \\
\hline & 3,15 & 3,96 & 6,68 & 3,87 & 3,76 \\
\hline
\end{tabular}

Tabla 7. Índices del balance cohesión-integración sociales (por categorías autóctono-inmigrante) de la población inmigrante asociada según el tipo de asociación en el que participan. Fuente: Elaboración propia.

Si introducimos también el lugar de residencia de los Alteri y el momento de inicio de la relación en el análisis (Figura 3) constatamos que los Egos que participan en asociaciones de servicios, además de tener menos Alteri autóctonos en sus redes y más en otros países, tienen una mayor proporción de Alteri residentes en el país de origen de Ego que el resto, mientras que en los otros tres tipos destaca la menor presencia de Alteri en el país de origen (menos incluso que entre los individuos que no participan). Es decir, las personas que participan en asociaciones de tipo "de servicios" tienen unas redes más dispersas ${ }^{6}$ y ancladas al país de origen, mientras que los que participan en otro tipo de asociaciones tienen unas redes más locales y movilizadas en el proceso migratorio. Si utilizamos las mismas categorías para observar la composición de la sub-red de Alteris que participan en la misma

\footnotetext{
${ }^{6}$ Molina, Bolíbar y Cruz (2011), en este mismo monográfico, trabajan ampliamente variedad de indicadores de la dispersión geográfica de las redes, pero en este caso al mencionar "dispersión" no se tiene en cuenta la distancia sino simplemente la localización de los Alteri en múltiples ubicaciones.
} 
asociación que Ego, vemos como los Alteri de asociaciones de tipo instrumental, además de tener más de autóctonos en esta sub-red (6,5\% más), estos también tienen más Alteri del mismo origen en el país de destino, especialmente de los conocidos en la sociedad de acogida (9,5\% más), a la vez que un poco más de Alteri de otros orígenes. En este caso, la naturaleza de los vínculos construidos en el campo asociativo se plasman en el conjunto de la red, teniendo así los participantes en asociaciones de tipo instrumental mayor capital social bridging $\mathrm{i}$ linking. De la misma forma, en el caso de las redes de los participantes en asociaciones relacionadas con la inmigración, tanto en la sub-red de Alteri con los que se comparte la actividad asociativa como en el conjunto de su red, se observan mas Alteri del mismo origen que Ego mantenidos - es decir, conocidos en el país de origen - ( $8,3 \%$ más) y menos Alteri de otros orígenes ( $7,5 \%$ menos).

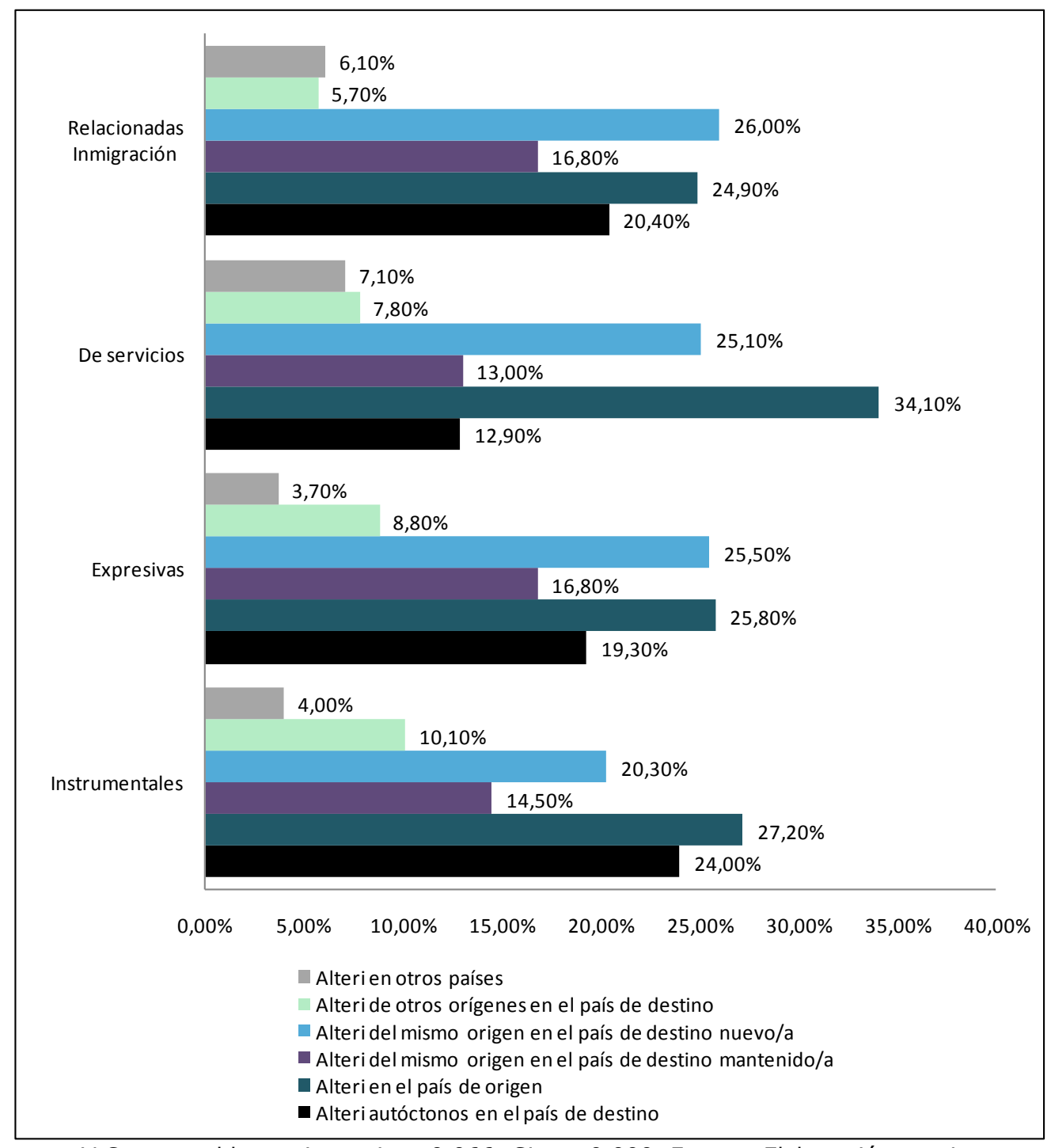

V Cramer tabla contingencia $=0,066 ;$ Sig. $=0,000$. Fuente: Elaboración propia.

Figura 3. Composición de las redes de la población inmigrante asociada en términos de origen y lugar de residencia, según el tipo de asociación en la que participan. 
Para acabar, el estudio del intercambio de apoyo social según el tipo de asociación en el que se participa nos muestra como aquellas personas que participan en asociaciones de servicios intercambian ayuda en un mayor número de ámbitos y ocasiones así como con una mayor proporción de Alteri (el $88 \%$ respecto al $76 \%$ de las instrumentales y el $80 \%$ de las expresivas y relacionadas con la inmigración). Si nos fijamos exclusivamente en aquellos Alteri con los que se comparte la actividad asociativa, observamos como hay diferencias entre el tipo de asociación en la que se participa y el tipo de apoyo que se intercambia con los Alteri de dichas asociaciones. Así, los Alteri de asociaciones instrumentales y relacionadas con la inmigración son los que intercambian un apoyo más instrumental (en relación al trabajo, vivienda, salud, educación, y servicios sociales y administrativos), mientras que los Alteri de asociaciones expresivas intercambian apoyo en menos ámbitos y sólo igualan e incluso superan las otras asociaciones en la proporción de Alteri con los que se intercambia el apoyo expresivo (problemas personales y otras situaciones).

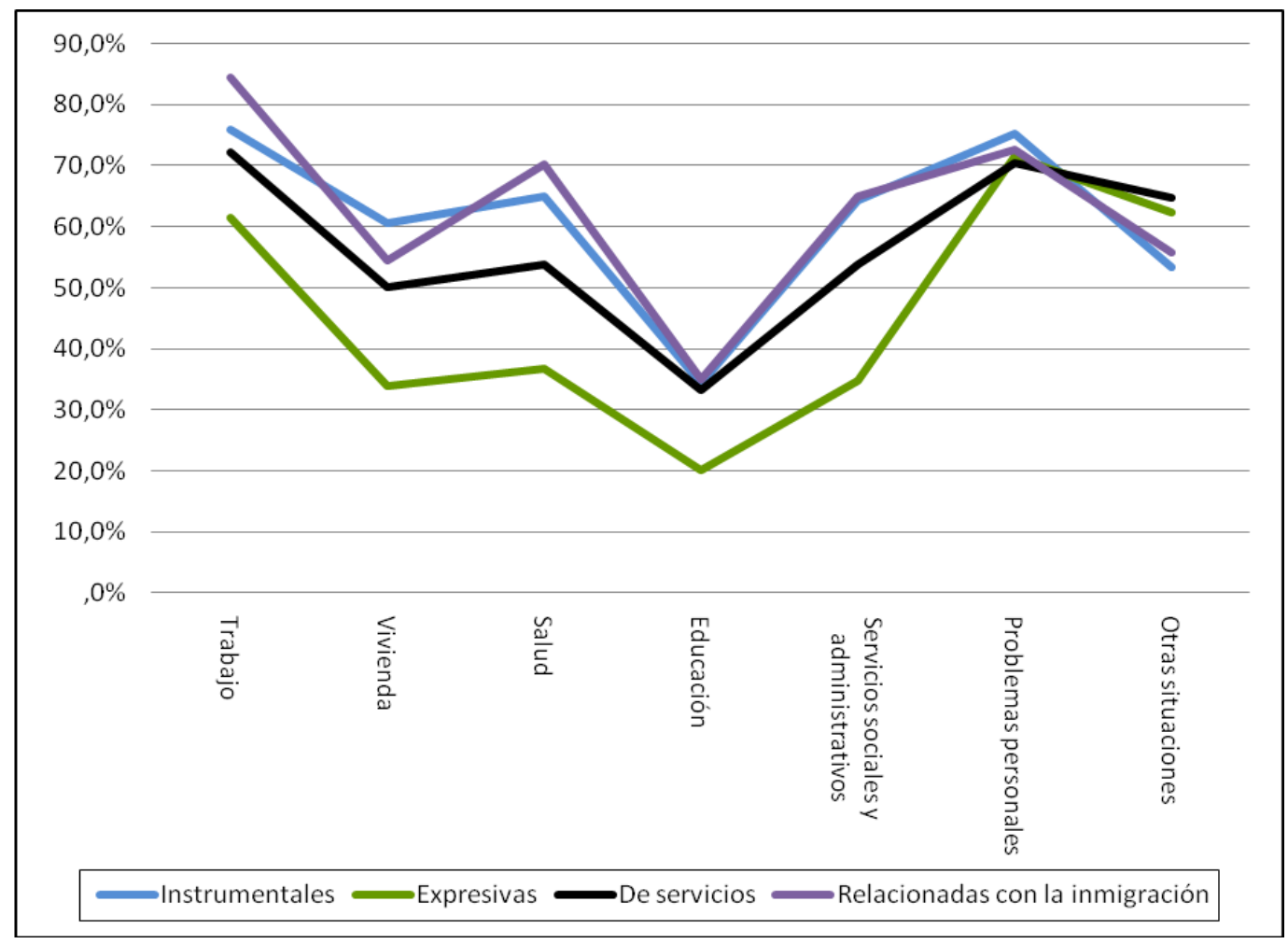

Figura 4. Tipo de apoyo intercambiado con los Alteri que participan en la asociación de Ego según el tipo de asociación en el que participan. Fuente: Elaboración propia. 


\section{Conclusiones}

Volvamos a las preguntas de la investigación. ¿Existen dinámicas relacionales propias en el tejido asociativo? ¿Facilitan estas dinámicas la integración de la población inmigrante, en el plano de las redes interpersonales? Una mirada panorámica a los datos presentados desvela que, aún con algunas especificidades propias, en general los individuos presentes en el campo asociativo no tienen unas redes sociales diametralmente diferentes a aquéllos que no participan en él. Podemos afirmar que se mantiene la tendencia a la cohesión y a la clausura de los grupos sociales de origen, aunque con una intensidad diferente.

En primer lugar, la población inmigrante asociada, aunque con menos inmigrantes del mismo grupo de origen "nacional" (ecuatorianos y marroquíes), dispone de un poco menos de autóctonos en sus redes y de más inmigrantes de otros orígenes, primando así las relaciones bonding y bridging, de cohesión y de vinculación social horizontal en las redes de los/as asociados/as más que en las del resto de la población. Sin embargo, el hecho de que quienes participan en asociaciones tengan menos autóctonos en sus redes no es debido al efecto directo de la participación en asociaciones, sino al contrario. En las redes de la población asociada, entre aquellos Alteri con los que se comparte la actividad asociativa hay una proporción mayor de autóctonos que entre los que no forman parte del ámbito asociativo. En segundo lugar, la introducción de la dimensión espacial nos permite observar que los vínculos generados en el campo asociativo son básicamente contactos locales ubicados en la sociedad de acogida. En tercer lugar, el análisis del lugar de inicio de las relaciones nos muestra que entre estos vínculos es mayor la proporción de inmigrantes del mismo origen que Ego conocidos en el país de destino que los conocidos en el país de origen con los que se mantiene la relación, creando así un entorno relacional más inmerso en la realidad inmigrante, con personas también inmigradas y en una situación vital semejante. En cuarto lugar, se aprecia como en la estructura del conjunto de las redes de las personas asociadas destaca la mayor presencia de subgrupos densos de Alteri conocidos entre sí. Finalmente, vemos como las personas que participan en asociaciones intercambian apoyo en un mayor número de ámbitos con el conjunto de sus Alteri, así como con los Alteri concretos que participan con Ego.

Sin embargo, el tejido asociativo no es una realidad homogénea. Como afirma Ariño, "este mundo asociativo es tremendamente heterogéneo y se halla internamente muy fragmentado y atomizado. Hoy por hoy, resulta imposible hablar de la existencia de un tercer sector, porque carece de una mínima articulación y 
coherencia" (2004: 89). También en lo que atañe a las redes sociales se observa esta diversidad; la participación en asociaciones no produce un efecto homogéneo sobre las redes de las personas que participan en ellas. Especialmente destacan los grupos y organizaciones caracterizadas como "de servicios" por ser las asociaciones que generan una realidad más independiente y diferente a las demás. Las personas inmigrantes que participan en ellas tienen unas redes más cohesivas y menos integradas, así como con una proporción mayor de Alteri residentes en el país de origen. Hay que señalar también que participan en ellas personas que hace menos tiempo que residen en la sociedad de acogida. Puede ser que uno de los motivos por los cuales se observan diferencias de género en el impacto que la participación en asociaciones produce en la composición de las redes de los inmigrantes, sea su diferente presencia en los diferentes tipos de asociación ${ }^{7}$. Se observa una tendencia a una mayor presencia de mujeres inmigrantes en asociaciones de tipo instrumental, mientras que se encuentran más hombres en asociaciones expresivas y en menor medida en asociaciones relacionadas con la inmigración.

En lo que respecta a la distinción entre asociaciones instrumentales y expresivas, bastante recurrente en la literatura, aunque hemos observado como se diferencian en algunas cuestiones -sobretodo en la naturaleza del tipo de apoyo social que se intercambia y en la intensidad de la cohesión de sus redes-, en este estudio no hemos constatado que las diferencias más relevantes dentro el panorama asociativo sean debidas a la distinción entre estos dos tipos de asociación. Existe una dificultad de inferir las dinámicas internas de sociabilización a partir de la única definición de la naturaleza de los objetivos y actividades de las asociaciones, cosa que también ha sido puesta en relieve por autores como Funes, 2006 -en Montero, Font y Torcal, 2006- o Lauer y Yan, 2010. Una motivación de tipo expresiva puede ser la que lleve a la participación en una asociación de tipo instrumental, mientras que una motivación instrumental inicial puede transformarse en una experiencia significativa en el aspecto expresivo. Además, nos hemos encontrado en nuestra muestra que en muchos casos se participa en múltiples asociaciones, tanto instrumentales como expresivas, lo que dificulta distinguir sus implicaciones en la variación de la composición de las redes. Aún así, consideramos que esta es una línea interesante de seguir explorando y en la que profundizar, también teniendo en cuenta otro tipo de diferencias generadoras de variabilidad en el interior del mundo asociativo, como por ejemplo la posición en redes inter-asociativas en el nivel meso-social.

\footnotetext{
$7 \mathrm{~V}$ de Cramer (asociación entre las variables "tipología participación en asociaciones" y "género de Ego" $^{\prime \prime}=0,207, \mathrm{sig}=0,000$.
} 
Volviendo al debate teórico sobre el efecto integrador o el efecto 'enclave' del asociacionismo sobre las redes de la población inmigrada, podemos afirmar que, si no tenemos en cuenta la participación en asociaciones de tipo "de servicios", aquellas personas que participan en asociaciones en general tienen unas redes un poco menos cohesivas que aquellas que no participan, sobretodo menos cohesivas con respecto al propio grupo de origen étnico o de nacionalidad de origen, y especialmente entre los miembros de asociaciones de tipo instrumental. La observación de la composición de la subred de Alteri que participan en la misma asociación a la que Ego pertenece nos indica como, si bien los datos validan que tendemos a participar con aquellas personas más homófilas a nosotros (Glanville, 2004) también es cierto que lo son en menor medida que el resto de Alteri. Además, los Alteri con los que se comparte la actividad asociativa son muy locales y cercanos geográficamente; fruto del desarrollo de un entorno relacional bastante nuevo y generado en buena parte en la sociedad de acogida.

Otra cuestión que merece la pena destacar es que el comportamiento relacional de la población inmigrante y autóctona no es muy diferente. Se observa también una gran cohesión en las redes de la población autóctona (en las cuales la presencia de personas inmigrantes es muy escasa), mayor incluso que entre la población inmigrante. Sin embargo, ésta disminuye si se observa la cohesión de los grupos estudiados existentes dentro de la categoría "población autóctona" (es decir, mayores de 55 años nacidos en el resto de España, jóvenes menores de 55 años nacidos en Catalunya, etc.), y, una vez más, se observa que el impacto de la participación en asociaciones sobre las redes de estos colectivos no es homogéneo.

En este estudio, metodológicamente ha resultado muy enriquecedor la introducción de las dimensiones espaciales y temporales en el estudio de la integración relacional de la población inmigrante, pues ha sido necesario conocer el lugar de residencia y el lugar y momento de inicio de la relación para obtener más pistas sobre las diferencias en la composición de las redes de aquellas personas que participan respecto las que no, lo que destaca la necesidad de una mirada más integral a la vez que más detallada sobre la estructura del capital social. No obstante quedan algunos interrogantes abiertos para resolver en futuras investigaciones. El impacto de la interacción entre las diferentes dimensiones y variables aquí tratadas como independientes sobre las redes, así como otros elementos relativos a la caracterización atributiva de los egos (como la nacionalidad de origen o el sexo) o de las asociaciones en las que participan, requieren un tratamiento futuro de los datos en más profundidad. Puede que una mirada más panorámica con aproximaciones de tipo cualitativo o con otros procedimientos de 
análisis de tipo multivariable permitan observar mejor la pluralidad de factores que influyen en las redes y la diferente importancia de las variables que en esta investigación exploratoria ya hemos señalado como claves.

Finalmente, resulta de interés hacer una reflexión sobre lo que explican los datos presentados. El entramado relacional refleja no sólo una problemática de los individuos en el nivel micro-social, sino que también muestran dinámicas colectivas fruto de la estructura social. En este contexto, el mundo asociativo (como estructura meso social que media entre las instituciones y los individuos y sus redes) reproduce la dinámica relacional cohesiva imperante en el territorio estudiado, aunque se observa también la gestación de un tímido cambio social en el plano de las redes de relación personal que fomenta. Será interesante ver su evolución en los próximos años a medida que madure el fenómeno migratorio y su incorporación en el tejido asociativo.

\section{Bibliografía}

Ariño, Antonio. (2004). "Asociacionismo, ciudadanía y bienestar social". Papers, revista de sociologia. Vol 74. Pp 85-110.

Baldassari, Delia; Diani, Mario. (2007). "The Integrative Power of Civic Networks". American Journal of Sociology. Vol. 113, No 3. Pp. 735-80.

Bekkers, R.; Völker, B.; Van der Gaag, M. \& Flap, H. (2008). "Social netowks of participants in voluntary associations". Pp. 184-205. En Lin, N. \& Erickson, B. (Eds.), Social Capital: Advances in Research. Oxford: Oxford University Press.

Casey, J. (1997). "Les associacions i la integració d'immigrants estrangers". Revista Catalana de Sociologia. No 6. Pp. 9-22.

Colectivo Ioé (1987) "Los inmigrantes en España". Documentación social, No 66.

Feld, S. (1982). "Structural determinants of similarity among associates", American Sociological Review, Vol. 47: 797-801.

Garreta, J. 1998. "Minories ètniques, associacionisme i integració sociocultural. Papers. Revista de Sociologia, núm. 56. Pp 197-230.

Garreta, J. Et al. 2008. "Associacionisme i acomodació de les persones immigrades d'origen africà a Catalunya". Col·leció ciutadania i immigració, de la Secretaria per a la Immigració de la Generalitat de Catalunya. núm. 1. 103-118. 
Glanville, Jennifer. (2004). "Voluntary Associations and Social Network Structure: Why organizacional location and type are important". Sociological Forum, Vol. 19, N03.

Hagan, Jacqueline. (1998). "Social Networks, Gender and Immigrant Incorporation: Resources and Constraints." American Sociological Review. Vol. 63, No 1. pp 55-67.

Lauer, S.R., Yan, M.C. (2010). "Voluntary Association Involvement and Immigrant Network Diversity", International Migrations, En prensa.

Lluch, A. M. (1999) "L'associacionisme dels immigrants", a AA.VV. (1999) Immigrants? Nous ciudadans. Barcelona: CIDOB.

Lozares C. (2003). Valores, campos y capitales sociales. En Redes, Revista hispana para el análisis de redes sociales. ISSN 157 9-0189. (Revista electrónica) Vol. 4. www.redes-sociales.net

Lozares, C. (2006). "Valores, Campos y Capitales sociales", Redes. Volumen especial, Bellaterra: Publicaciones UAB, pp. 51-89

Lozares, C.; J. M. Verd, P. López-Roldán, J. Martí \& J. L. Molina (2011). "Cohesión, Vinculación e Integración sociales como formas de Capital social", REDES-Revista hispana para el análisis de redes sociales, vol. 20 \#1 (http://revistaredes.rediris.es), enviado.

Lozares, C.; J. M. Verd, P. López-Roldán, J. L. Molina, M. Bolíbar \& I. Cruz (2011). "El análisis de la Cohesión, Vinculación e Integración sociales en las encuestas Egonet" ", REDES-Revista hispana para el análisis de redes sociales, vol. 20. \#4 (http://revista-redes.rediris.es), enviado.

Marsden, Peter V. (1990). "Network Diversity, substructures, and opportunities for contact". En Calhoun, C., Meyer, M., Scott, W.R. (Eds). Structures of Power and Constraint: Papers in Honor of Peter M. Blau. Pp 397-410. Cambridge: Cambridge University Press.

Martí, J., Lozares, C. (2008). "Redes organizativas locales y capital social: Enfoques complementarios desde el análisis de redes sociales". Portularia. Revista de Trabajo Social. Universidad de Huelva. , Vol. 7, pp. 461-480. 
Martínez García, M. F., García Ramírez, M. et al. (2001). "Una tipología analítica de las redes de apoyo social en inmigrantes africanos en Andalucía." REIS. Vol. 95, Pp.99-125.

Maya Jariego, I., Martínez, M. F., y García, M. (1999). Cadenas migratorias y redes de apoyo social de las mujeres peruanas en Sevilla. Demófilo: Revista de Cultura Tradicional de Andalucía, 29, 87-105.

Maya Jariego, I. (2006). Mallas de paisanaje: el entramado de relaciones de los inmigrantes. En Pérez Pont, J. L. (Ed.). Geografías del desorden. Migración, alteridad y nueva esfera social, 257-276. Universidad de Valencia: Valencia.

McPherson, J.M., \& L. Smith-Lovin. (1987) "Homophily in voluntary organizations: status distance and the composition of face-to-face groups", American Sociological Review, Vol. 52. Pp. 370-79.

Miguel Luken, V. \& Tranmer, M. (2010) "A multilevel analysis of the personal support networks of immigrants to Spain". Social Networks. Vol. 32 No 4. Pp 253262.

Molina, J.L., Lerner, J., Gómez Mestres, S. (2008). "Patrones de cambio de las redes personales de inmigrantes en Cataluña". Redes. Revista Hispana para el análisis de las redes sociales, Vol. 15, \#4 http://revista-redes.rediris.es.

Molina, J.L., Bolíbar, M., \& Cruz, I. (2011). "La dispersión geográfica de las redes personales: cálculo y significado". REDES-Revista hispana para el análisis de redes sociales, vol. 20 (http://revista-redes.rediris.es), enviado.

Montero J.R., Fontj., Torcal, M. (eds.). 2006. Ciudadanos, asociaciones y participación en España. Madrid: Centro de Investigaciones Sociológicas.

Morell Blanch, A. 2005. "El papel de las asociaciones de inmigrantes en la sociedad de acogida: cuestiones teóricas y evidencia empírica". Migraciones, 17. Pp 111-142.

Prakash, Sanjeev. \& Selle, Per. (Eds.). (2004). Comparative perspectives on Civil Society, Participation and Governance. New Delhi: Sage Publications.

Putnam, Robert. (1993). Making Democracy Work:Civic Traditions in Modern Italy. Princeton: Princeton University Press.

Putnam, Robert. (2000). Bowling Alone: The Collapse and Revival of American Community. New York: Simon and Schuster. 
Putnam, Robert. (2007). "E Pluribus Unum: Diversity and Community in the Twenty-first Century". Scandinavian Political Studies. Vol.30, No 2, Pp. 137-174.

Popielarz, Pamela, \& McPherson, J.Miller. (1995). "On the Edge or in between: Niche position, Niche overlap, and the duration of voluntary association memberships". American Journal of Sociology. Vol 101. Pp 698-720.

Ribas, Natalia (2003). "Dona i immigració". En Aubarell, G. (dir), Gestionar la diversitat. Reflexions i experiències sobre les polítiques d'immigració a Catalunya. Barcelona: Temed. Pp. 170-184.

Rodríguez-García (2010). "Beyond Assimilation and Multiculturalism: A Critical Review of the Debate on Managing Diversity". International Migration and Integration. Vol 11 (3). Pp. 251-271.

Rotolo, Thomas. (2000). "Town heterogeneity and affiliation: A multilevel análisis of voluntary associations membership". Sociological Perspectives. Vol. 43, No 2. Pp 271-289.

San Martín, Josep. (2009). "Catalunya, paradís de la societat civil? Explicacions i conseqüències de la participació en associacions". Barcelona: Memoria del Doctorado en Ciencia Política, Departament de Ciencia Política i de Dret Públic. UAB [No publicado].

Sipi, Remei. (2000). "Las asociaciones de mujeres, ¿agentes de integración social?". Papers, revista de sociologia. No 60. Pp 355-364. 